\title{
Exploiting No-SQL DB for Implementing Lifelog Mashup Platform
}

\author{
Kohei TAKAHASHI, Shinsuke MATSUMOTO, \\ Sachio SAIKI, and Masahide NAKAMURA \\ Kobe University, \\ 1-1 Rokkodai-cho, Nada-ku, Kobe, Hyogo 657-8501, Japan \\ \{koupe@ws.cs, shinsuke@cs, sachio@carp, masa-n@cs\} .kobe-u.ac.jp
}

\begin{abstract}
To support efficient integration of heterogeneous lifelog service, we have previously proposed and implemented a lifelog mashup platform consisting of the lifelog common data model (LLCDM) and the lifelog mashup API (LLAPI) to access the standardized data. The LLCDM has standardized columns which is application-independent. And it has application-specific data (i.e. JSON format text of API response of a lifelog service) in the <content> column as a plain text. But because the LLCDM repository is implemented using the relational database, we can't access to the <content> column data directory, and select out a particular field of it via the LLAPI. To cope with these problems, we exploited the lifelog mashup platform with the document-oriented No-SQL database MongoDB for the LLCDM repository. And, we conduct a case study developing an application of retrieving Twitter's posts involving URLs.
\end{abstract}

Keywords: lifelog, mashup, no-SQL, mongoDB, web services, api

\section{Lifelog Mashup}

\subsection{Lifelog Services and Mashups}

Lifelog is a social act to record and share human life events in open and public form [1]. Various lifelog services currently appear in the Internet. By them, various types of lifelogs are stored, published and shared. For example, Twitter[2] for delivering tweets, Flickr[3] for storing your photos, foursquare[4] for sharing the "check-in" places, blog for writing diary.

Mashup is a new application development approach that allows users to aggregate multiple services to create a service for a new purpose [5]. For example, integrating Twitter and Flickr, we may easily create a photo album with comments (as tweets).

Expecting the lifelog mashup, some lifelog services are already providing APIs, which allow external programs to access the lifelog data. However, there is no standard specification among such APIs or data formats of the lifelog. Figure 1(a) is a data record of Twitter, describing information of a tweet posted by a 
user "koupetiko" on 2013-07-05. Figure 1(b) shows a data record retrieved from SensorLoggingService, representing a various sensor's values of user "koupe" on 2013-07-05. We can see that data schema of the two records are completely different. Although both are in the JSON (JavaScript Object Notation), there is no compatibility. Note also that these records were retrieved by different ways by using proprietary APIs and authentic methods. Thus, the mashup applications are developed in an ad-hoc manner, as shown in Figure 2.

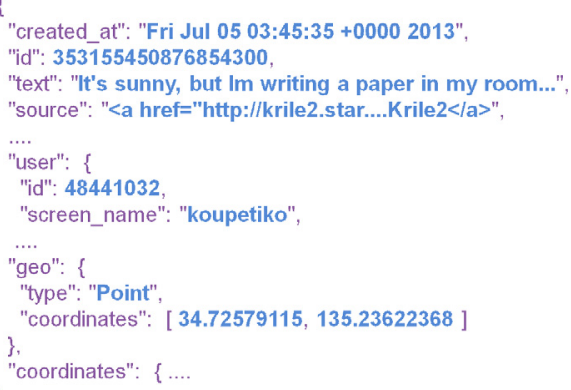

(a) A data record of Twitter

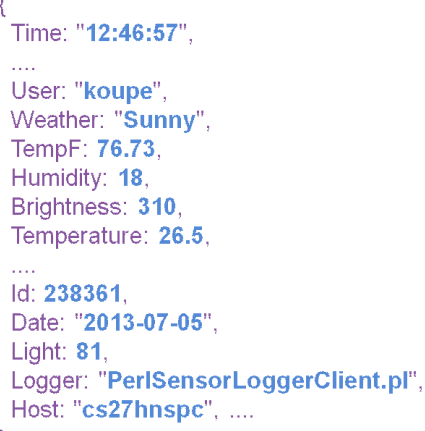

(b) A data record of Sensor Logging Service

Fig. 1. Data of two different lifelog services

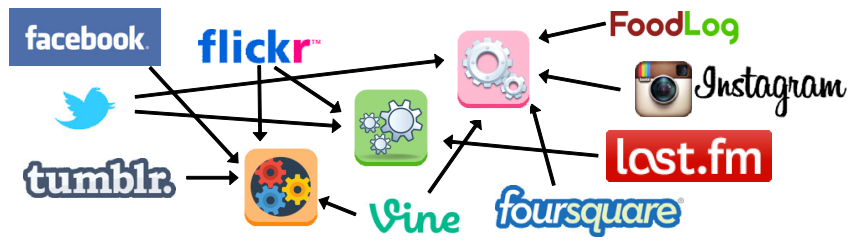

Fig. 2. Conventional Approach of Lifelog Mashup

\section{Previous work}

\subsection{Lifelog Mashups Platform}

To support efficient lifelog mashup, we have proposed a lifelog mashup platform [6] previously . The platform consists of the lifelog common data model (LLCDM) and the lifelog mashup API (LLAPI), as shown in Figure 3. 


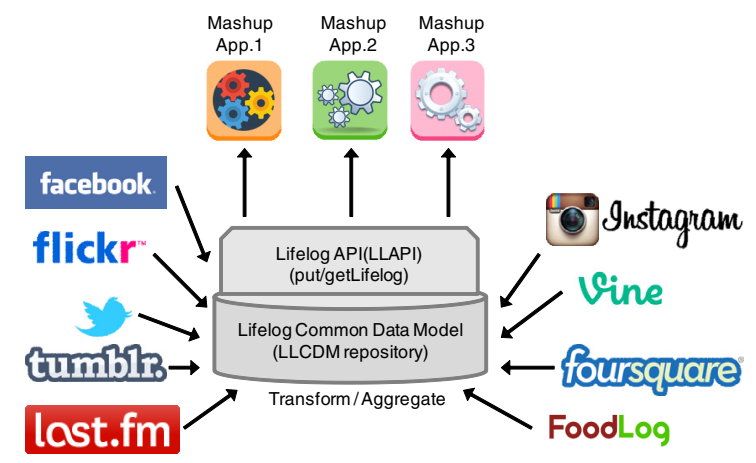

Fig. 3. Proposed Lifelog Mashup Platform [6]

Table 1. Common data schema of LLCDM

\begin{tabular}{|c|c|c|c|}
\hline \multicolumn{2}{|c|}{ perspective data items } & Description & Instance \\
\hline \multirow[t]{3}{*}{ WHEN } & $\langle$ date $\rangle$ & Date when the log is created (in UTC) & $2013-07-05$ \\
\hline & $\langle$ time $>$ & Time when the log is created (in UTC) & $12: 46: 57$ \\
\hline & <epoch> & UNIX Time (sec) when the log is created (in UTC) & 1373028417 \\
\hline \multirow[t]{3}{*}{ WHO } & <user> & Subjective user of the log & koupe \\
\hline & $<$ party $>$ & Party involved in the log & shinsuke_mat \\
\hline & <object> & Objective user of the log & masa-n \\
\hline \multirow[t]{5}{*}{ WHERE } & $\langle$ latitude $>$ & Latitude where the log is created & 34.72631 \\
\hline & <longitude> & Longitude where the $\log$ is created & 135.23532 \\
\hline & $<$ altitude > & Altitude where the $\log$ is created & 141 \\
\hline & $<$ address > & Street address where the $\log$ is created & 1-1, Nada, Kobe \\
\hline & $\langle$ name> & place name where the log is created & Kobe University \\
\hline \multirow[t]{2}{*}{ HOW } & <application> & Service/application by which the log is created & Flickr \\
\hline & <device> & Device with which the $\log$ is created & Nikon D7000 \\
\hline \multirow[t]{2}{*}{ WHAT } & $\langle$ content> & Contents of the $\log$ (whole original data) & <photo id=".." owner=".." title=".." \\
\hline & $\langle$ ref_schema> & URL references to external schema & http://www.flickr.com/services/api/ \\
\hline WHY & $\mathrm{n} / \mathrm{a}$ & $\mathrm{n} / \mathrm{a}$ & $\mathrm{n} / \mathrm{a}$ \\
\hline
\end{tabular}

\subsection{Lifelog Common Data Model}

The data stored in heterogeneous lifelog services are transformed and aggregated in the LLCDM, which is an application-neutral form among the lifelog services.

Table 1 shows the data schema of the LLCDM. We arranged the data items which lifelog records should prepare from the viewpoints of what, why, who, when, where and how. Then, we defined the "common data store" which does not depend on specific service and application.

\subsection{Lifelog API}

The LifeLog Mashup API $(L L A P I)$ is for searching and retrieving lifelog data conforming to the LLCDM. The following shows an API that returns lifelog data matching a given query. Using getLifeLog(), heterogeneous lifelogs can be accessed uniformly without proprietary knowledge of lifelog services. 


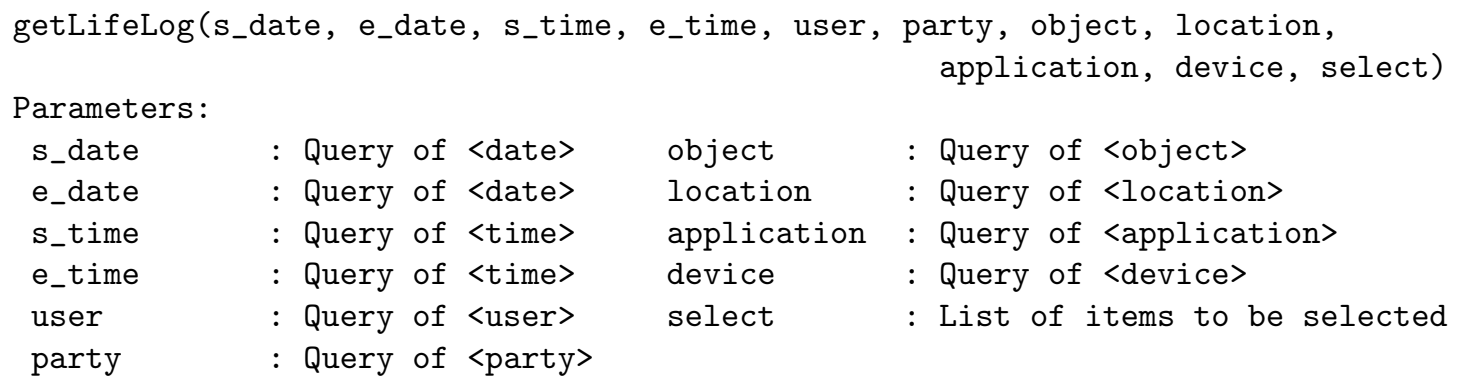

This is implemented as a query program wrapping an SQL statement. The following SQL statement is published by the getLifelog() method.

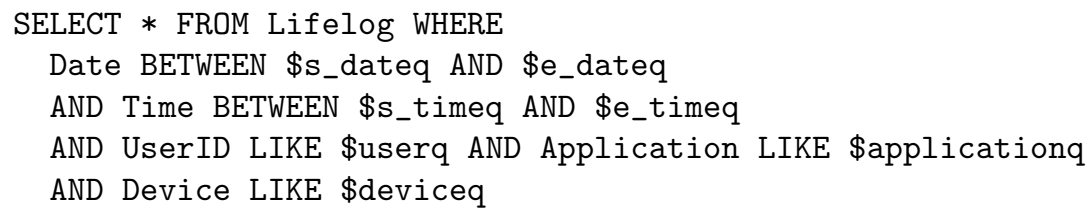

LLAPI is published as Web service (REST, SOAP) and can be invoked from various platforms.

\subsection{Limitations in Previous work}

The limitation is the accessibility to the data in <content> column. In [6], we have implemented the LLCDM repository using relational database (i.e. MySQL), and deployed the LLAPI with Web service. In this way, the data of <content> column is intentionally left uninterpreted within the LLCDM. Actually, it is stored as plain text in spite of structured data (e.g. xml, json). So, we can't access specified fields in the <content> column of lifelog data by queries., although, it is possible to some extent by using text matching. Nevertheless, we can't "select" the specified fields of <content> column just like other fields of the LLCDM. For example, it is impossible to retrieve lifelogs of content.entities.urls having any items, or to retrieve only date, time, content.user.screen_name columns of each lifelogs, etc. Thus, application developers have to parse <content> data in their applications.

The fewer required items in <content>, this brings more wasteful development cost, traffic, and the worse performance.

\subsection{Research Goal and Approach}

Our interest here is to achieve flexible query to the data of <content> column.

To achieve this goal. We put all the lifelog data in a document-oriented NoSQL database, instead of having a relational Database. Using this, we aim to flexible access to the data of <content> column. But the data except <content> column should be fixed according to the LLCDM schema. So, we re-engineer the LLAPI as facade of the database, and it to be used as SQL-like. 


\section{Exploiting No-SQL DB for Implementing lifelog Mashup Platform}

\subsection{Using MongoDB for Lifelog Mashup}

To achieve the goals in Section 2.5, we introduce the MongoDB to manage the LLCDM repository (See Figure 3.). MongoDB is a schema less document oriented database developed by 10 gen and an open source community [7]. MongoDB was designed to provide both the speed and scalability of key-value datastores as well as the ability to customize queries for the specific structure of the data [8]. In MongoDB, the terms "collection" and "document" are used instead of "table" and "row" in SQL. It has the following features.

Pros

\section{P1: Document-Oriented Storage}

MongoDB stores documents as BSON (Binary JSON) objects, which are binary encoded JSON like objects. BSON supports nested object structures with embedded objects and arrays like JSON does [7].

\section{P2: Full Index Support}

MongoDB indices are explicitly defined using an ensureIndex call, and any existing indices are automatically used for query processing [9].

\section{P3: High Availability, Easy Scalability}

MongoDB supports automatic sharding, distributing documents over servers [9]. And it supports replication with automatic failover and recovery, too. Also MongoDB supports MapReduce.

\section{Cons}

\section{C1: No Transaction}

MongoDB has no version concurrency control and no transaction management [10]. However, atomic operations are possible within the scope of a single document.

\section{C2: No JOIN}

MongoDB doesn't support joins. So, some data is denormalized, or stored with related data documents to remove the need for joins [11].

To use MongoDB for the Lifelog Mashup Platform, P1 and P2 match highly the our purpose, because in the LLCDM repository, data in <content> column has various structure and various kinds of elements depends on their application. But, it can't force the data in defined formats or types of each columns, and the columns except <content> must be in the defined format in the LLCDM. So, it is necessary to take measures for this policies. By doing this, we will achieve SQL-like searching on all columns of the LLCDM. Also, P3 is very favorable for our future work which is capacity for bigdata.

On the other hand, about $\mathrm{C} 1$, most of operations are read and write on the Lifelog Mashup Platform. So, we consider there is no special trouble with C1.

Next, about C2, in data modeling of MongoDB, when entities have "contains" relationships, it is recommended to embedding the child in the parent. 


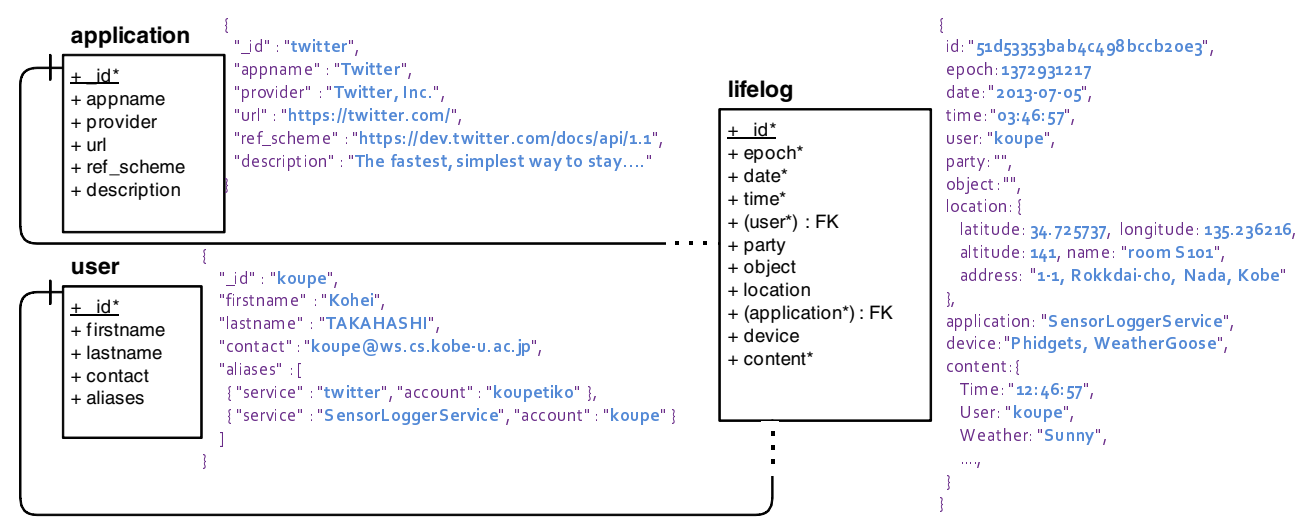

Fig. 4. ER diagram for the LLCDM repository

When entities have one-to-many relationships, it is recommended to embedding or referencing. The embedding is recommended in the case of one-to-many relationships where the "many" objects always appear with or are viewed in the context of their parent. In the new implementation, we comply with this.

\subsection{Implementing LLCDM with MongoDB}

Considering the above, we have re-designed the data model of the LLCDM for using MongoDB. Figure 4 shows the proposed ER diagram of the LLCDM. A box represents an entity (i.e., table, but it's called collection in MongoDB.), consisting of an entity name, a primary key, foreign key, and attributes. We enumerate instances beside each entity in JSON format to support understanding. A line represents a relationship between entities, where $+-\cdots$ denotes a reference relationship. The underlined attributes of entities are primary keys. The attributes in brackets of entities in a relationship are foreign keys. An attribute with a asterisk is NOT Nullable. The diagram consists of the following three collections.

(a) application: In this collection, we manage the applications from which we retrieve the lifelog data record. The application information is useful to identify the types of lifelog data. Therefore, we consider it efficient to manage it in a separate collection. The attributes of this collection are ID, Application name, Provider, URL, Reference URL and Description.

(b) user: This collection manages the user information, consisting of ID, User name, Contact information, Aliases to application accounts. The reason why we provide this collection is that the user information is commonly attached in various lifelog data, and is one of the most frequently used information in the mashup. Also, Aliases achieves to associate various applications' accounts 
or multiple accounts in the same service with an actual person. This was one of the remaining subjects of previous work [6].

(c) lifelog: This is the main collection of the LLCDM. All retrieved lifelog data consisting every item of the LLCDM is stored. As mentioned in Section 2.2 and Table 1, lifelog has each data item arranged from the viewpoints of what, why, who, when, where and how. In this regard, <ref_schema> is nothing in lifelog because it is in application.

\subsection{Implementing LLAPI with MongoDB}

We have implemented putLifelog() method and getLifelog() method. Since MongoDB is a No-SQL database, the LLAPI must take a role of a part of a relational database (i.e., data type check, data format check, key constraint, and so on.). So, the putLifelog() method as a facade of the LLCDM repository. Calling the putLifelog( $)$ method with parameters corresponding attributes of the entity, the parameters are validated based on the LLCDM and insert to MongoDB. Thus, objects inserted via putLifelog() method are guaranteed their formats in the LLCDM schema definition.

Furthermore, SQL-like database retrieval must be kept as same as in previous work. And to achieve more flexible retrieving, we have implemented the getLifelog() method as follows.

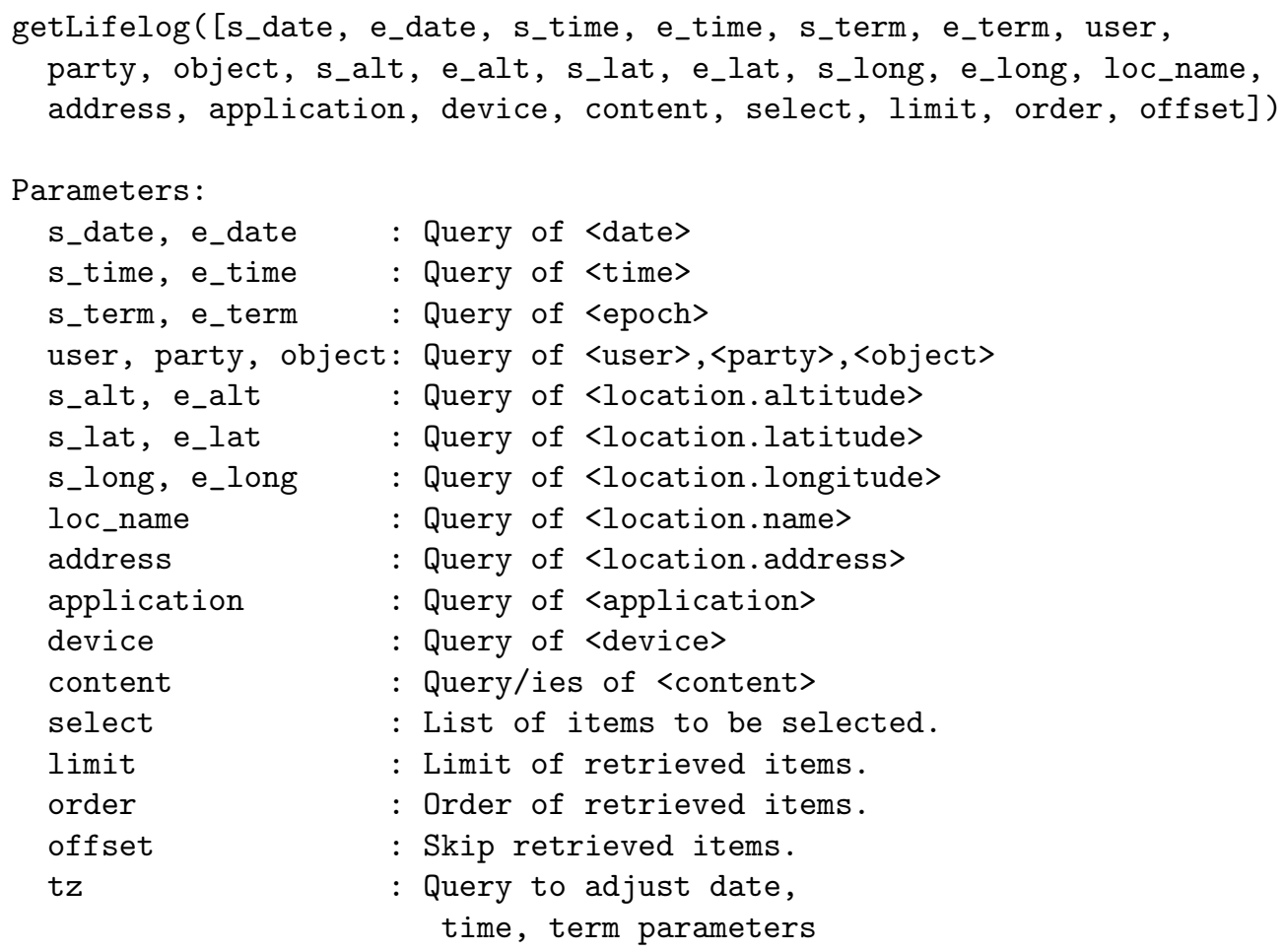


Table 2. Comparison of Functions

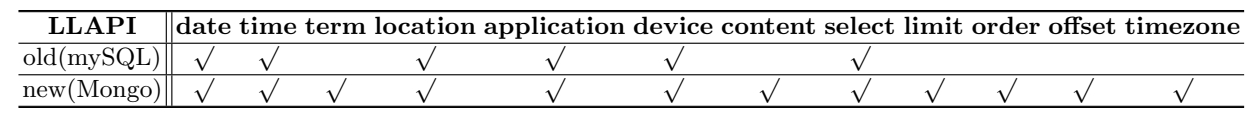

And this method publishes the following MongoDB Query Language. In the case of null parameters, the corresponding query would be nothing (i.e. All parameters were null, getLifelog() returns all lifelogs in the LLCDM.). Also, to publish the query, date, time, term parameters are adjusted to UTC time based on tz parameter. The $\$$ contentq is an array of queries to <content> column (e.g. "content.temperature \$gte 25 , content.humidity $\$ 1$ t $40 ")$. The $\$$ selectq is an array of items to be selected (e.g. "date, time, content.temperature").

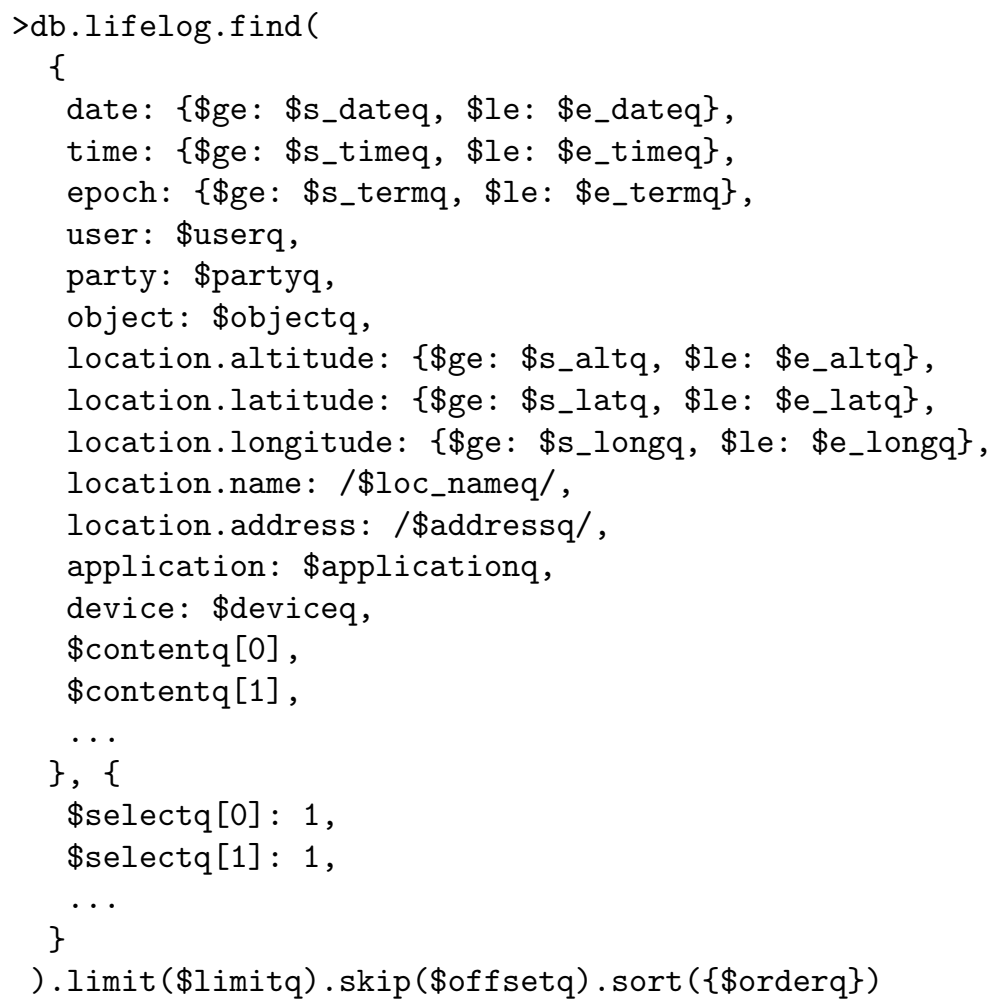

Also, Table 2 shows the comparison of functions between the old prototype and the new implementation. In this table, a check mark means an available parameter of each LLAPI. As shown Table 2, the new implementation of the LLAPI is more flexible than the old prototype. 
We implemented the LLAPI in the Java language. We used Morphia[12] ORmapper for marshaling tuples into objects. And for validating objects, we used Java Validation API (Hibernate Validator (JSR303) Reference Implementation for Bean Validation.[13]).

Furthermore, we used JAX-RS (Jersey: JAX-RS (JSR 311) Reference Implementation for building RESTful Web services [14].) to provide RESTful API.

Now that the LLAPI can be accessed by the REST Web service protocol. Figure 5 shows a response of the LLAPI. We can see that a Twitter data record describing a tweet posted by a user "koupetiko" on 2013-05-01 has been retrieved.

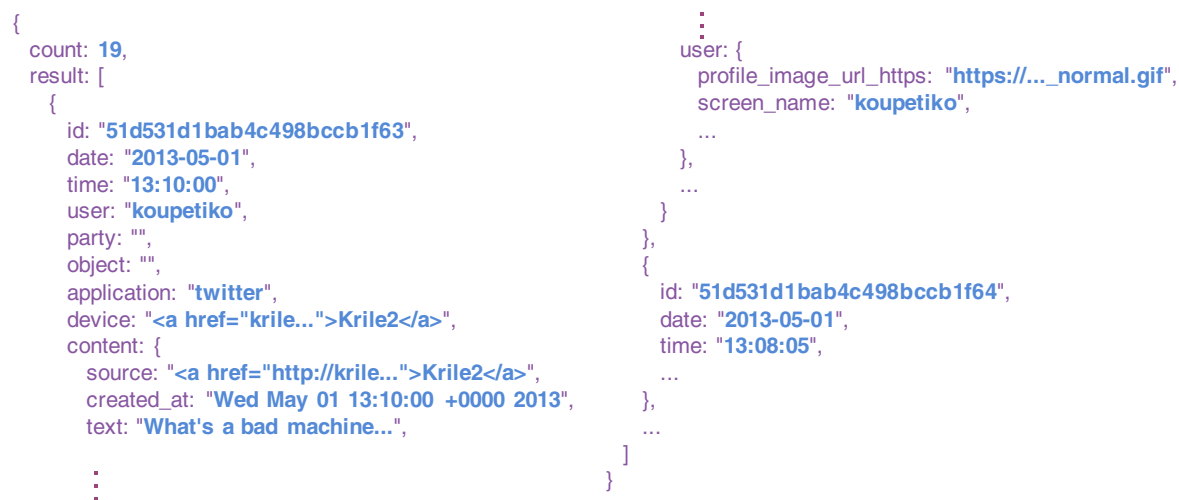

Fig. 5. a response of getLifelog

\section{Case Study}

Using the proposed method, we develop a practical Web application My Tweeted URLs. The application My Tweeted URLs provides a simple viewer of one's tweets involving urls and their web pages' thumbnails. And the thumbnails link their web pages. For retrieving and processing the lifelogs of tweets, we have used jQuery. Also, for obtaining thumbnails, we have used public API of WordPress.com. The total lines of code is just 96, including the code of html and jQuery. Figure 6 is the screenshot of this application.

We have implemented this application so easily because we only had to obtain the thumbnails and show the tweets of retrieved lifelogs from the LLCDM. This process is the essential process of this application.

If we use old prototype, to implement this, we would have to do some wasted jobs. First, we retrieve all lifelog data in the target period from the LLCDM, and parse their <content> column data in JSON format text to JavaScript object. 


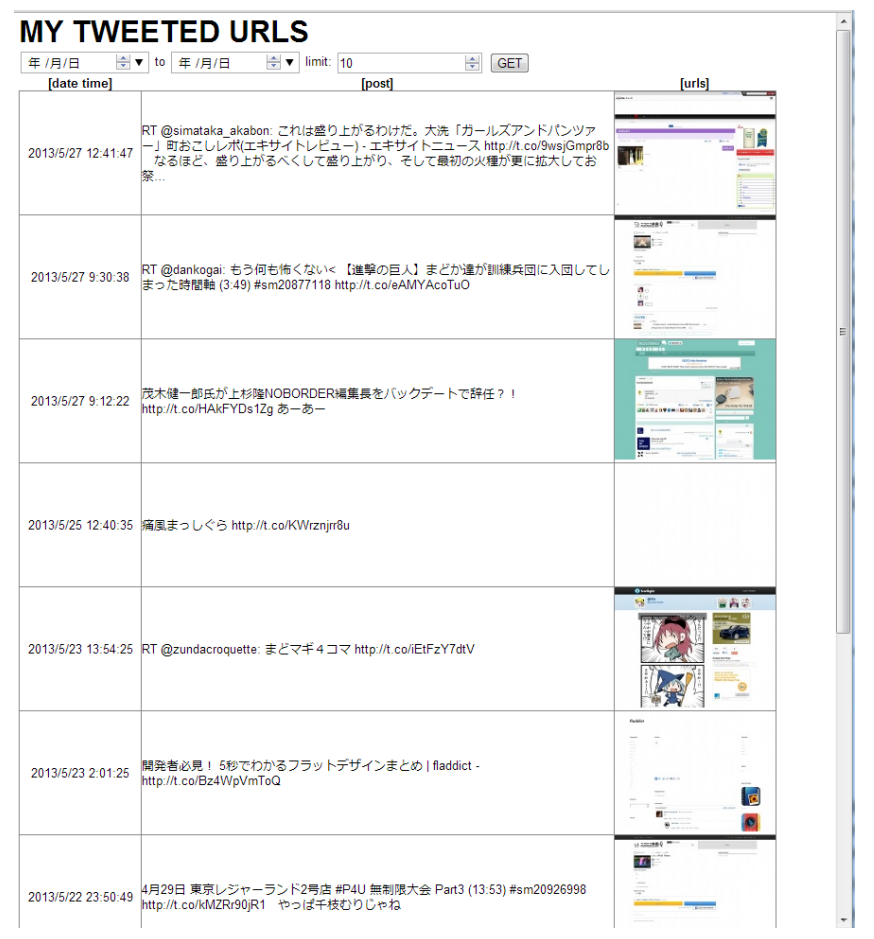

Fig. 6. Screenshot of "My Tweeted URLs"

After that, check each data if its tweet has "http". At last, we change over to essential process of obtaining the thumbnails and showing it with tweets.

\section{Conclusion}

In this paper, to improve the accessibility of the data, we have re-engineered the lifelog mashup platform [6], consisting of the LLCDM (LifeLog Common Data Model) and the LLAPI (LifeLog Mashup API). Specifically, we exploited the No-SQL database for the LLCDM repository to achieve flexible query to <content> column data. As a case study, we developed the lifelog application "My Tweeted URLs", and we made sure that the proposed method would reduce the development effort and complexity of source code of the lifelog application.

Our future work includes performance improvement, and evaluate the capacity for bigdata. We also plan to study potentials of lifelog mashups for business and education.

Acknowledgments This research was partially supported by the Japan Ministry of Education, Science, Sports, and Culture [Grant-in-Aid for Scientific Research (C) (No.24500079), Scientific Research (B) (No.23300009)]. 


\section{References}

1. Trend Watching .com: Life caching - an emerging consumer trend and related new business ideas http://trendwatching.com/trends/LIFE_CACHING.htm.

2. Twitter: http://twitter.com/.

3. Flickr: http://www.flickr.com/.

4. foursquare: http://foursquare.com/.

5. Lorenzo, G.D., Hacid, H., young Paik, H., Benatallah, B.: Data integration in mashups. Volume 38., ACM (2009) 59-66

6. Shimojo, A., Matsuimoto, S., Nakamura, M.: Implementing and evaluating life-log mashup platform using rdb and web services. In: The 13th International Conference on Information Integration and Web-based Applications \& Services (iiWAS2011). (December 2011) 503-506

7. Padhy, R.P., Patra, M.R., Satapathy, S.C.: Rdbms to nosql: Reviewing some nextgeneration non-relational databases. International Journal of Advanced Engineering Science and Technologies 11(1) (2011) 15-30

8. Bunch, C., Chohan, N., Krintz, C., Chohan, J., Kupferman, J., Lakhina, P., Li, Y., Nomura, Y.: Key-value datastores comparison in appscale. (2010)

9. Cattell, R.: Scalable sql and nosql data stores. SIGMOD Rec. 39(4) (May 2011) $12-27$

10. Milanović, A., Mijajlović, M.: A survey of post-relational data management and nosql movement

11. 10gen, Inc.: Mongodb http://www.mongodb.org/.

12. Morphia: https://github.com/mongodb/morphia.

13. Hibernate-Validator: http://www.hibernate.org/subprojects/validator.html.

14. Jersey: http://jersey.java.net/. 